general, a size distribution of workers which precisely matches the distribution of size of available resource items is not to be expected. Their argument relies heavily on the concept of "fidelity cost", which assumes that an abrupt increase in the number of individuals in a given class creates an energy cost to the colony. This is an interesting idea, but seems rather difficult to evaluate empirically, in view of the necessarily arbitrary way in which the fidelity cost is introduced into the models. Chapter 7 is concerned with foraging strategies of workers. Chapter 8 is a useful discussion of the role of

optimisation theory in evolutionary biology, and Chapter 9 is a brief survey of unsolved problems. As the authors make clear, both here and in the preface, the major factors determining the evolution of the detailed features of caste ratios and behavioural flexibility remain largely unknown. Nevertheless, their use of quantitative models highlights the problems very clearly and should prove very stimulating to research in this area.

Brian Charlesworth is Lecturer in Biology at the University of Sussex, Brighton, UK.

\title{
Affinity chromatography
}

An Introduction to Affinity Chromatography. By C.R. Lowe. Pp. 253. (North-Holland: Amsterdam, New York and Oxford, 1979.) \$29.25; Dfl60.

THIS volume is part of a series on laboratory techniques. The first four chapters consist of an introduction to chromatography, and accounts of basic concepts, chemistry and techniques of affinity separations. Chapter 5 is a selection of applications (protein-enzyme area), and a separate chapter covers regulatory macromolecules and more complex structures (although how antibody-antigen systems fit this category escapes me). The last two chapters are concerned with analytical applications and special techniques (very useful). The book covers most of the earlier work in the field: 9 references out of the 240 are 1977 or later.

The author has many thoughtful remarks to make for users of affinity chromatography. Rather than present a flawless up-to-the-minute review, $\mathrm{Dr}$ Lowe provides a valuable set of guidelines which should inspire rather than weary the reader. Because the technique is developing so rapidly some parts of the book are incomplete. Thus, it is now clear that triazine dyes are widely used as affinity ligands, their main advantage being the combination of capacity and selectivity. A slightly misleading comment on p455 suggests that all albumins will bind to Cibacron Blue columns when this property refers to human albumin only.

In the next edition, I would like to see some of the following recent advances discussed: the receptor purification advocated by Baulieu, involving alteration of the molecular weight of a protein using soluble dextran-ligand conjugates, electrophoretic desorption of high affinity biospecific adsorbents, light-reversible affinity ligands, and high performance affinity chromatography.

The sections on ligand concentration, temperature effects, the importance of ligand preassembly and the concept of group specific affinity matrices did not do justice to the author's work in these areas. I would also have liked to see a warning of the toxicity of $s$-trichlorotriazine (cyanuric chloride).

Pride of place in accounts of affinity chromatography goes to the now famous use of immobilised oxamate to adsorb lactate dehydrogenase in the presence of $\operatorname{NAD}(\mathbf{H})$. Dr Lowe's book leaves this bastion of theoretical preconception unassaulted, although Wood et al. have convincingly shown (1977) that replacing $\mathrm{CNBr}$ with triazine chemistry abolishes the biospecificity of the oxamate column.

Apart from the above minor criticisms, I found the balance between commercials, laboratory methods (for which Dr Lowe has an obvious flair) and theory enjoyable and well thought out.

P.D.G. Dean

P.D.G. Dean is Senior Lecturer in the Department of Biochemistry at the University of Liverpool, UK.

\section{Biological semiconduction}

Dielectric and Electronic Properties of Biological Materials. By R. Pethig. Pp.376. (Wiley: Chichester, UK, and New York, 1979.) $£ 15$.

THE possibility that electronic energy bands exist in certain solid-state biological systems, playing a role in energy migration (Möglich and Schön; Jordan 1938) and electron transfer (Szent-Györgyi, 1941) has been around for over 40 years. During this time there has occurred a small but steady amount of theoretical and experimental progress on biological semiconduction, that is, on the electron transfer problem. Earlier work tended to show the conductivities were too low for biological significance, but with passage of time, the effects of hydration, and of chargetransfer, in inducing appreciable electronic conduction in proteins, all go towards increasing the prospects of a role of semiconduction in certain biological processes. The recent demonstration of high conductivity in reduced cytochrome $c_{3}$ by
Inokuchi and coworkers, is another step in the same direction. Dr Pethig's book is aimed at introducing newcomers from a variety of disciplines to the field, and these are to be welcomed, as the need to increase the ratio of facts to speculations is rather urgent.

Very sensibly Pethig's first three chapters deal successively with dielectric theory, the intrinsic dielectric properties of biopolymers, and the effects of solvation on the dielectric properties. In these he established the basic solution properties, and then goes on in his fourth chapter to the effects of water on the solid-state behaviour, dealing with X-ray studies and water adsorption. In his fifth chapter Pethig outlines interfacial dielectric phenomena, the Maxwell-Wagner effect and its developments. Although this treatment is clear, I believe it could usefully have been a greater length, as the separation of Maxwell-Wagner from dipolar and hopping loss mechanisms is a fundamental problem in this field. Per contra, the sixth chapter dealing with dielectrophoretic studies is mainly of interest in connection with preparative separation methods, and is slightly off the main theme of the book. Chapter 7 deals with biological membranes and tissues, with brief notes on black lipid membranes, and includes three pages on the sometimes controversial effects of electromagnetic fields. With the final two chapters, 8 and 9, we come to the main part of the book, in which the author's own research interests are to be found. Chapter 8, on electrons, energy levels and energy bands, provides the basic background for crystalline and amorphous semiconductors, and includes an account of band gap and band width calculations for biopolymers, from the pioneer work of M.G. Evans and J. Gergely (1949) to the present. Chapter 9 sets out the experimental data on semiconductivity, Hall effects, charge-transfer interactions, piezoelectric effects and so on for proteins, DNA and other biological molecules. Photoconductivity is mentioned, but rather too briefly in view of its possible importance in vision and photosynthesis. Chapters 8 and 9 , however, could scarcely be comprehensive in the space available, and Pethig's approach has been to take a few selected topics in depth. So, for example, there is a thoughtful appraisal of the effects of adsorbed water on conductivity, and an account of Pethig's own work on the methylglyoxal-protein system, in connection with Szent-Gyorgyi's recent suggestions concerning carcinogensis.

I believe this book gives an attractive introduction to its field. Mathematics are kept within the bounds necessary for a general readership, and in spite of the author's engineering background the emphasis is always on molecules rather than equivalent circuits. It is logically arranged, reads easily, and there is an ample supply of references. As a personal preference, I think a chapter summarising 